\title{
Hydrocortisone-modulated Immune Response and Altered Amino Acid Pool in Vitro
}

\author{
Peter N. Uchakin ${ }^{\mathrm{a}, \mathrm{c}}$, Scott M. Smith ${ }^{\mathrm{b}}$, Olga N. Uchakina ${ }^{\mathrm{a}}$
}

\begin{abstract}
Background: Dietary deficiencies, from macro- to micronutrients, are often associated with dysfunction of the immune system. The aim for this study was to assess changes in amino acid metabolism and immune response in a modeled "stress" environment.

Methods: Heparinized blood samples from healthy donors were collected by venipuncture. Peripheral blood mononuclear cells were isolated and then incubated with a mixture of LPS and PHA in RPMI-1640 medium. Cell cultures were treated with hydrocortisone at physiological $\left(10^{-8} \mathrm{M}\right)$ and "stress" $\left(10^{-6} \mathrm{M}\right)$ concentrations. Surface expression of $\mathrm{CD} 25$ by the $\mathrm{CD}^{+}$lymphocytes was analyzed by flow cytometry. Levels of the cytokines IL-2, IFN- $\gamma$, IL-4, and IL-10 were measured with ELISA. Quantification of IL-2- and IFN- $\gamma$ - secreting T cells was performed in cell cultures treated with PMA, ionomycin, and brefeldin A.
\end{abstract}

Results: The number of $\mathrm{CD}^{+} \mathrm{CD} 25^{+}$cells was significantly lower in cultures treated with $10^{-6} \mathrm{M}$ of hydrocortisone than in control cultures. Treatment of cell cultures with $10^{-8} \mathrm{M}$ hydrocortisone significantly increased levels of IFN- $\gamma$ and IL-10, but not IL-2 and IL-4. Treatment with $10^{-6} \mathrm{M}$ of hydrocortisone significantly suppressed secretion of all studied cytokines. Treatment with $10^{-8} \mathrm{M}$ hydrocortisone produced a consistent unidirectional effect on amino acids in the supernatant medium: increased concentrations of almost all amino acids. Treatment with $10^{-6} \mathrm{M}$ hydrocortisone significantly decreased the level of asparagine

Manuscript accepted for publication October 5, 2011

${ }^{a}$ Mercer University School of Medicine, Division of Basic Medical Sciences, Macon, GA 31207, USA

bNASA Johnson Space Center, Nutritional Biochemistry Laboratory, 2101 NASA Road 1, Houston, TX 77058, USA

${ }^{\mathrm{c}}$ Corresponding author: Peter N. Uchakin, Mercer University School of Medicine, 1550 College Street, Macon, GA 31207-0001, USA.

Email: uchakin_pn@mercer.edu

doi: $10.4021 /$ jem $49 w$ while it increased levels of serine and tyrosine compared to control cultures.

Conclusions: Data suggest that the stress-dose of cortisol modulated amino acid metabolism in mitogen-stimulated immunocompetent cells in vitro. Also, observed differences in amino acid concentrations between cultures suggest that supplementation with low doses of endocrine mediators may create a more physiological environment for culturing PBMCs.

Keywords: Immunity; Cytokines; Cortisol; Stress

\section{Introduction}

Dietary deficiencies, from macro- to micronutrients, are often associated with dysfunction of the immune system. Leukocytes, lymphocytes, and all lymphocyte subset counts were lower in subjects with hypocaloric diets than in controls [1]. Malnutrition resulted in a shift in the ratio between naive and memory T-cell subsets in mice [2] compared to eucaloric controls. In contrast, dietary supplementation with omega-3 polyunsaturated fatty acids led to activation of immune responsiveness, as they activated gene expression of interferon (IFN)- $\gamma$ and interleukin (IL)-12 in mice infected with live Listeria monocytogenes [3].

The role of amino acids in nominal function of any physiological system cannot be overstated. The role of several amino acids (such as arginine, cysteine, ornithine, serine, and histidine) in immune mechanisms is well documented [4-13]. Close interactions between amino acid metabolism, the endocrine system, and the immune stress response have been demonstrated in vitro, in animal models, and in clinical studies, including studies with burn patients and septicemia patients. Animal studies demonstrated alterations in amino acid metabolism in response to mitogenic challenges. Arginine supplementation increased mitogen-induced proliferation of mouse splenocytes [14]. In a pig model, intramuscular lipopolysaccharide (LPS) administration resulted in significant increases in plasma concentrations of alanine, cystine, glycine, hydroxyproline, phenylalanine, and serine, 
but decreases in the levels of glutamine, isoleucine, leucine, threonine, and tyrosine [15]. Activation of porcine lymphocytes with phytohemagglutinin (PHA) accelerated $\mathrm{Na}^{+}$-dependent uptake of the amino acids proline and methionine [16]. Dietary supplementation with arginine, glutamine, cysteine, and taurine enhanced immune responsiveness among critically ill patients (reviewed in [17]) and glutamine recovered immune suppression in pigs infected with Escherichia coli $[18]$.

In human trials, it has been shown that parenteral nutrition with glutamine dipeptide had beneficial effects on the immune response in post-surgical patients [19]. Six months of nutritional supplementation significantly increased the percentage of mature $\mathrm{T}$ cells in peripheral blood of elderly subjects [20]. The rates of uptake of cationic amino acids were increased in cells from patients with septic shock [21]. Glutamine supplementation attenuated the immunosuppression and decreased the rate of infection in burn patients [22].

The hypothalamic-pituitary-adrenal (HPA) axis [2326], and especially corticosteroids, appear to be a significant factor in homeostatic regulation of the immune response to stress. Lower mitogen-induced production of IL-2 and reduced lymphocyte sensitivity to glucocorticoids were associated with greater concentrations of salivary cortisol in caregivers than in non-caregivers [27]. Negative correlations were observed between urinary cortisol levels and the percentages of several T-cell subsets after dexamethasone treatment [28]. Changes in the sensitivity of lymphocytes to glucocorticoids were observed in septic shock patients [29]. Additionally, it was found that hydrocortisone and dexamethasone can reactivate the Epstein-Barr virus genome in latently infected lymphoblastoid cells [30].

These data strongly suggest a close connection between immune and metabolic stress responses, but the details of this relationship are yet to be clarified. The main objective of this study was to aid in this clarification by assessing changes in amino acid metabolism along with mitogen-stimulated production of immune mediators and markers under normal conditions as well as in an HPA axis-mediated "stress" environment mimicked by hydrocortisone.

\section{Materials and Methods}

\section{Subjects and samples}

This study was approved by the Institutional Review Board of Mercer University School of Medicine. Seven - to 10 - mL blood samples from healthy donors $(n=9)$ were collected by venipuncture into heparinized Vacutainer tubes (BD, Franklin Lakes, NJ, USA). The blood was mixed with a double volume of Hanks balanced salt solution, layered over $20 \mathrm{~mL}$ of Ficoll-Paque, and centrifuged to isolate peripheral blood mononuclear cells (PBMCs).

\section{Cell cultures and assessment of immune responsiveness}

Isolated PBMCs at a final concentration of $1 \times 10^{6}$ cells $/ \mathrm{mL}$ were incubated with $10 \mu \mathrm{g} / \mathrm{mL}$ of LPS from Escherichia coli 026:B6 and $5 \mu \mathrm{g} / \mathrm{mL}$ of PHA in RPMI-1640 medium supplemented with L-glutamine $(2 \mathrm{mmol} / \mathrm{L})$ and gentamicin sulfate $(50 \mathrm{mg} / \mathrm{mL})$ (Sigma, St. Louis, MO, USA) for up to 72 hours at $37{ }^{\circ} \mathrm{C}$ and $95 \%$ humidity. Surface expression of IL-2 receptor $\alpha$-subunit (CD25) by T cells was analyzed at 24 hours; secretion of IL-2, IFN- $\gamma$, IL-4, and IL-10 by PBMCs was measured at 48 hours; and lymphocyte proliferation was assessed at 72 hours of incubation. Additional 4-hour cell cultures in which PBMCs were activated with $25 \mathrm{ng} / \mathrm{mL}$ of PMA, $1 \mu \mathrm{g} / \mathrm{mL}$ of ionomycin in the presence of $10 \mu \mathrm{g} / \mathrm{mL}$ of brefeldin A were established to quantify T-cell subsets that secrete IL-2 and IFN- $\gamma$.

Quantification of $\mathrm{CD}^{+} \mathrm{T}$ cells expressing surface CD25 was performed by direct immunofluorescence labeling of cell surface CD3 receptor antigen with mouse antihuman monoclonal antibodies conjugated to fluorescein isothiocyanate (FITC), phycoerythrin (PE), and peridinin chlorophyll protein (PerCP) (BD, Franklin Lakes, NJ, USA), using procedures recommended by BD. Thresholds for $\mathrm{CD}_{25^{+}}$cell enumeration were set using isotype control antibodies. PerCP-labeled anti-CD4 or anti-CD8 monoclonal antibodies were used for quantification of the cytokineproducing T-cell subsets. PBMCs were permeabilized and labeled with a mixture of PE-conjugated anti-IL-2 and FITCconjugated anti-IFN $-\gamma \mathrm{mAbs}$ according to the procedure recommended by BD. Samples were analyzed on an EPICS ${ }^{\circledR}$ XL flow cytometer (Beckman Coulter, Brea, CA, USA).

The concentrations of IL-2, IFN- $\gamma$, IL-4, and IL-10 were assayed in the cell cultures with commercial enzyme-linked immunosorbent assay (ELISA) kits (R\&D Systems, Minneapolis, MN, USA) and read on the Multiscan MS Plate Reader (Labsystems, Helsinki, Finland) according to the manufacturer's recommendations.

\section{Cortisol modulation of immune responsiveness}

Cell cultures were treated with the water-soluble hormone hydrocortisone (HCS) (Sigma, St. Louis, MO) at concentrations of $10^{-8} \mathrm{M}$ (HCS-8) and $10^{-6} \mathrm{M}$ (HCS-6) to study effects of the HPA axis on the immune stress response in vitro. The $10^{-6} \mathrm{M}$ concentration reflects plasma cortisol concentrations documented in runners after exposure to the physical stress of running a marathon [31], as well as after the i.v. injection of Solucortef (hydrocortisone Na succinate) to mimic the endocrine HPA stress response in healthy volunteers [32]. The $10^{-8} \mathrm{M}$ concentration of HCS reflects physiological levels of unbound plasma cortisol in resting healthy individuals [33, 34]. 


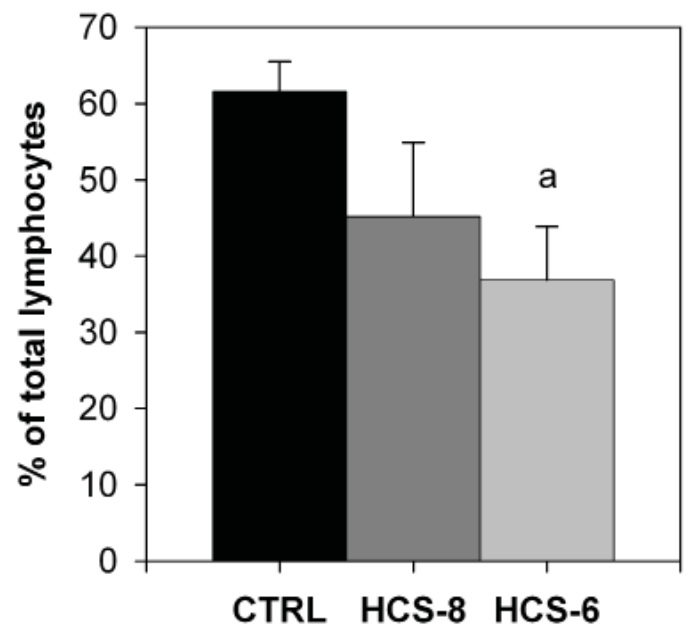

Figure 1. Effect of $10^{-8} \mathrm{M}$ and $10^{-6} \mathrm{M}$ hydrocortisone (HCS-8 and HCS-6, correspondingly) on the number of $\mathrm{CD}^{+} \mathrm{CD} 25^{+}$cells in 24-hour cell cultures stimulated with a PHA + LPS mixture for 24 hours. Values are means \pm S.E.M. a: Significantly $(P<$ 0.05) different from control (CTRL).

\section{Assessment of amino acids and their metabolites}

Amino acid analysis of the samples of cell culture medium was performed using a Hitachi L-8800 amino acid analyzer, as described previously [35]. This instrument uses ionexchange chromatography with post-column ninhydrin derivatization and visible colorimetric detection to allow the analysis of amino acids in a variety of physiological samples. The samples were prepared for amino acid analysis by precipitating protein with a solution of sulfosalicylic acid (SERAPREP ${ }^{\mathrm{TM}}$ ). The protein-free filtrate was adjusted to a $\mathrm{pH}$ of 2.2 and then placed in a refrigerated sample tray.
Depending on their dissociation characteristics, the amino acids are differentially eluted from the column with a series of lithium buffers of increasing $\mathrm{pH}$ and ionic strength, and further positioned by increasing the column temperature during the run. Ninhydrin, mixed continuously with the column eluent, reacts in a highly specific manner with the separated amines to form colored products, the intensity of which is proportional to the concentration of the amino acid present. The 148-minute method used with the Hitachi L-8800 amino acid analyzer allows the quantification of up to 44 identifiable compounds, which represent the free amino acid pool.

\section{Statistical analysis}

Data were analyzed using SigmaStat statistical software (Systat Software Inc., Point Richmond, CA, USA). The Kolmogorov-Smirnov test was used to determine normality of the data. Within-subject ANOVA with All Pairwise Multiple Comparison Procedures (Holm-Sidak method), and Friedman RM ANOVA on ranks were used to compare results from the different cell cultures. A value of $\mathrm{P} \leq 0.05$ was considered significant.

\section{Results}

The number of $\mathrm{T}$ lymphocytes expressing the IL-2 receptor $\alpha$-subunit (CD25) after LPS + PHA challenge was significantly lower in cultures treated with HCS-6 than in control cultures, but the difference was not significant for cultures treated with $10^{-8} \mathrm{M}$ hydrocortisone (Fig. 1).

No noteworthy changes occurred in the number of cytokine-secreting T-cell subsets in cell cultures activated with PMA + ionomycin in response to treatment with either

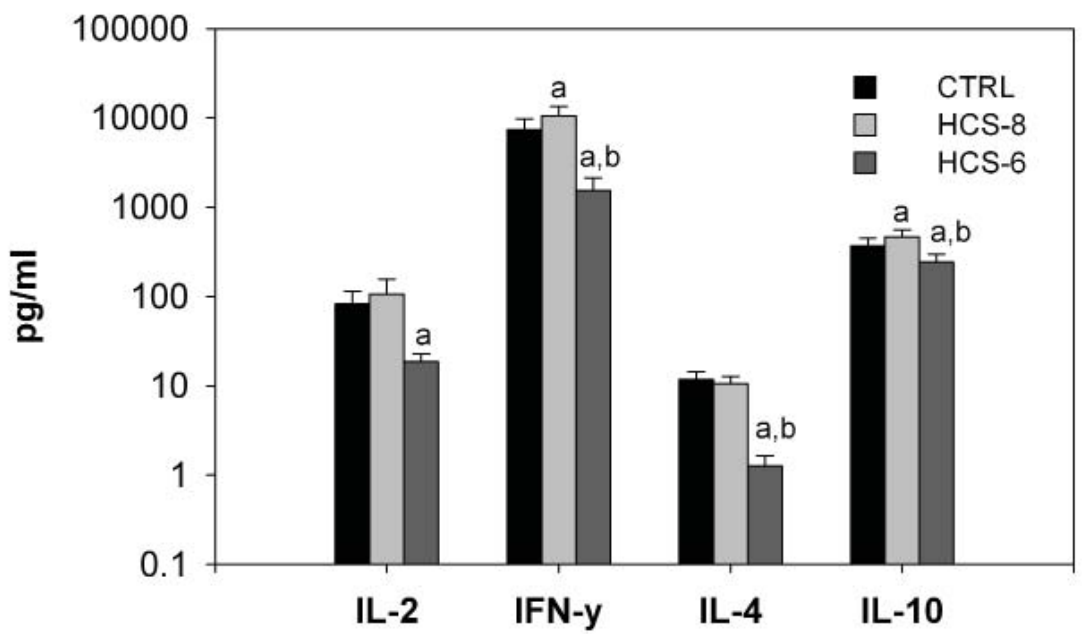

Figure 2. Effects of $10^{-8} \mathrm{M}$ and $10^{-6} \mathrm{M}$ hydrocortisone (HCS-8 and HCS-6, correspondingly) on cytokine secretion in 24-hour cell cultures stimulated with a PHA + LPS mixture. Values are means \pm S.E.M. a: significantly different $(P<$ 0.05) from CTRL culture. b: significant difference $(P<0.05)$ between HCS-8 and HCS-6 cultures. 

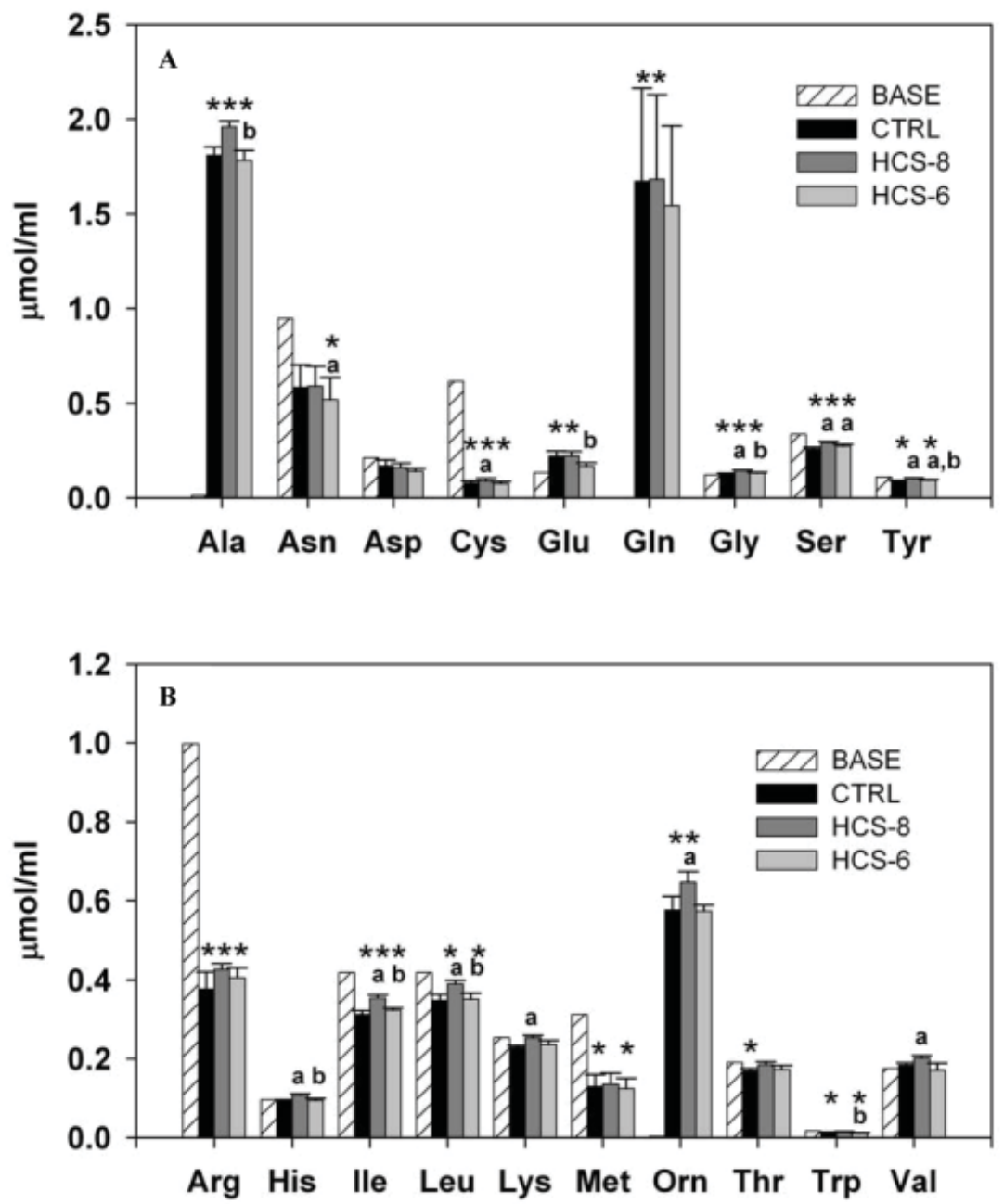

Figure 3. Effect of $10^{-8} \mathrm{M}$ and $10^{-6} \mathrm{M}$ hydrocortisone (HCS-8 and HCS-6, correspondingly) on the concentration of essential (A) and nonessential (B) amino acids in 24-hour cell cultures stimulated with a PHA + LPS mixture. Values are means \pm S.E.M. Data are presented in comparison with the baseline (BASE) concentration measured in cell cultures before incubation. *, **, ${ }^{* *}$ Statistically significant differences $(P<0.05)$ between cultures. *Significantly different from BASE; a: significantly different from CTRL; b: significant difference between HCS-8 and HCS-6 cultures.

dose of hydrocortisone (data not shown). An increase of borderline statistical significance $(\mathrm{P}=0.048)$ in the numbers of IL-2-secreting $\mathrm{CD}^{+}$lymphocytes occurred in the cell culture treated with $10^{-8} \mathrm{M}$ hydrocortisone.

Treatment of cell cultures with $10^{-8} \mathrm{M}$ hydrocortisone significantly increased secretion of IFN- $\gamma$ and IL-10, but not IL-2 and IL-4 (Fig. 2). Secretion of all studied cytokines was significantly suppressed by the "stress" concentration $\left(10^{-6}\right.$ M) of hydrocortisone compared to control cultures (Fig. 2).

Culture of PBMCs with hydrocortisone affected the concentration of the majority of the studied amino acids and their metabolites compared to their basal level in the culture medium. Exceptions were observed only for aspartic acid, histidine, lysine, valine (Fig. 3), and urea (data not shown). The power of the statistical analysis, with $\alpha=0.05$, exceeded
0.8 for this computation for the majority of amino acids, the only exceptions being lysine and threonine, for which it was 0.75 and 0.64 , respectively.

Treatment of cell cultures with $10^{-8} \mathrm{M}$ hydrocortisone produced a consistent unidirectional effect, with increased concentrations, compared to controls, of almost all amino acids in the supernatant medium. However, a statistically significant effect of this concentration was documented only for cysteine, glycine, serine, tyrosine, histidine, isoleucine, leucine, lysine, ornithine, and valine (Fig. 3A, B). Treatment with the stress dose of hydrocortisone significantly decreased the level of asparagine while increasing the levels of serine and tyrosine compared to controls. Also, statistically significant differences between HCS- 8 and HCS- 6 cultures were observed in the concentrations of alanine, glutamic 
acid, glycine, tyrosine, histidine, isoleucine, leucine, and tryptophan.

\section{Discussion}

Data collected in this study demonstrate that the cortisol-mediated stress response is able to affect the immune response to mitogen challenge in vitro, both quantitatively and qualitatively. A decrease in the number of mitogen-responsive $\mathrm{T}$ lymphocytes as well as downregulation of cytokine secretion can be considered immunosuppressive. Simultaneously we observed significant changes in amino acid metabolism in cell cultures of mitogen-stimulated immunocytes. It is important to note that all of the immune function variables studied (such as CD25 expression and cytokine secretion) depend on protein synthesis, and thus require gene activation with consequent translation and transduction.

A substantial body of literature supports the notion that most stress-induced immune reactions are mediated by neuroendocrine mechanisms, and cortisol is one of the most common candidates for stress mediators in immune alterations. In general, cortisol is considered to be an immunosuppressive agent. However, it is important to consider that cortisol exhibits its immunosuppressive and anti-inflammatory properties only in the higher physiological (and/or pharmacological) doses. In the present study, we used $10^{-6} \mathrm{M}$ hydrocortisone to mimic the HPA stress response with consequent release of cortisol. Similar concentrations were observed in the plasma samples of runners after they had completed a marathon [31]. In our previous studies [36], we used this concentration as a test model to show changes in the sensitivity of immunocompetent cells to endogenous cortisol in humans subjected to a stressful environment. Also, similar concentrations were used previously to demonstrate immunomodulatory effects of corticosteroids in vitro [36-40]. Indeed, in the present study, the "stress level" $\left(10^{-6} \mathrm{M}\right)$ of HCS significantly suppressed cytokine secretion and decreased the number of T cells expressing CD25 receptor. The finding that the number of cytokine-secreting T-lymphocyte subsets exhibited no response to the hydrocortisone treatment could be attributed to the specificity of the pathway of activation by the PMA + ionomycin mixture. In a parallel study, we found significant (over 30\%) suppression of ${ }^{3} \mathrm{H}$-thymidine uptake in cell cultures treated with the stress dose of hydrocortisone and activated by LPS + PHA but not in those activated by PMA + ionomycin (data not shown). The PMA + ionomycin mixture is a very potent activator that may lead to the release of very high concentrations of cytokines IL-2 and IFN- $\gamma$ by lymphocytes [41]. Thus, the doses of hydrocortisone that were used in this study may not have been sufficient to significantly affect the number of cytokine-secreting T-cell subsets in our setting. Also, shorter duration of the cell culture could be another factor in the observed "nonrespon- siveness" by the cytokine-secreting T lymphocytes to $10^{-6} \mathrm{M}$ hydrocortisone.

The lower concentration of hydrocortisone used in this study $\left(10^{-8} \mathrm{M}\right)$ reflects the physiological level of unbound cortisol in plasma $[33,34]$. As such, it represents a more physiological in vitro environment than the unsupplemented CTRL culture. Treatment of PBMCs with $10^{-8} \mathrm{M}$ hydrocortisone significantly increased secretion of IFN- $\gamma$ and IL-10, and increased the number of T cells producing IL-2 and IFN- $\gamma$. These observations are consistent with a number of previous findings. Specifically, Reid et al showed that hydrocortisone at $10^{-6} \mathrm{M}$ and $10^{-7} \mathrm{M}$, but not at $10^{-8}$ $\mathrm{M}$, increased bone resorption in neonatal mouse calvaria [42]. Feinstein et al evaluated the effect of hydrocortisone at doses of $10^{-9}-10^{-5} \mathrm{M}$ on the IL-1-induced secretion of the granulocyte-macrophage colony-stimulating factor (GMCSF) by primary human bronchial epithelial cells, and demonstrated that hydrocortisone at a concentration 5 times its physiological concentration $\left(5.0 \times 10^{-8} \mathrm{M}\right)$ produced $50 \%$ inhibition of GM-CSF secretion [33]. Also, HCS at $10^{-8} \mathrm{M}$ significantly increased both the protein and mRNA levels of IL- $1 \alpha$ in Pam 212 cells through the AP-1 and NF- $\kappa$ B activation pathways $[37,40]$.

It appears that observed differences in results primarily reflect different research models, and, perhaps more importantly, regulatory properties of cortisol that depend on physiological state. For example, while in most cases a "stress" concentration of hydrocortisone causes immune suppression, it has been demonstrated that hydrocortisone at $10^{-6} \mathrm{M}$ can significantly upregulate IL-2, IL-4, IL-7, and IL-13 receptor mRNAs in stimulated B lymphocytes in vitro [43]. These results once again demonstrate multiple functionality of cortisol and the importance of recognizing it as an immunomodulatory rather than an immunosuppressive agent. Moreover, it might explain why humoral immunity is less suppressed by stress factors, than cell-mediated immune reactions.

Evidence also exists that the adrenal stress response can alter amino acid metabolism. Cortisol administration upregulates enzymatic activities, which lead to production of ornithine and proline from arginine, as well as the formation of glutamate, alanine, aspartate, ornithine, citrulline, and proline from glutamine in porcine enterocytes [44]. On the other hand, in vitro study showed that preincubation of human trophoblastic cells with cortisol significantly upregulated the transfer rate of methyl amino isobutyric acid and increased SNAT2 expression, which suggests that cortisol is able to stimulate amino-acid transport system A on the molecular level [39]. It is important to note that both of these processes affect basic protein synthesis, such as cytokine production in immunocompetent cells. Despite the fact that higher doses of cortisol were used in these studies, these data and our findings allow us to suggest that cortisol-mediated alterations in amino acid metabolism and transmembrane transport can be 
significant mechanisms for regulation of the immune stress response. Yet, it may also be possible that cortisol-mediated alterations in cytokine production lead to altered anabolic and/or catabolic mechanisms. Indeed, in the most recent studies Jones et al demonstrated that even physiological concentrations of the pro-inflammatory cytokines are able to stimulate the activity of AA system A in vitro [45].

\section{Conclusion}

Mechanisms of observed changes need to be studied further. Nevertheless, our data allow us to conclude that selected amino acids (specifically availability of the Asn, Cys, Gly, Ser, Tyr, Arg, Ile, Leu, and Met) may be important for the adequate immune responsiveness in humans subjected to a chronic stressful environment. Furthermore, considering methodological differences our data are in accord with the fact that the same amino acids were affected during bacterial infection [15], while administration of some of them recovers immune suppression $[17,18]$. This allows us to speculate that alteration of amino acid metabolism is the significant (if not central) factor of the stress-induced secondary immune deficiencies. Also, observed differences in amino acid concentrations between CTRL and HCS- 8 cultures suggest that supplementation with low doses of endocrine mediators such as cortisol may create a more physiological environment for culturing PBMCs.

\section{Acknowledgments}

The authors thank Dr. Francis C. Dane for his help with statistical analysis, and Dr. Jane Krauhs for help with editing the manuscript. This work was supported in part by the MEDCEN Community Health Foundation and through Cooperative Agreement NCC 9-58 with NASA, NSBRI grant NFPR00205 suppl. The funding source had no role in the design and conduct of the study, in the collection, analysis, and interpretation of the data, or in the preparation, review, or approval of the manuscript.

\section{Conflicts of Interest}

None.

\section{References}

1. Lopez-Varela S, Montero A, Chandra RK, Marcos A. Effect of the diet on the nutritional status of ballerinas: immunologic markers. Nutr Hosp. 1999;14(5):184-190.

2. Woodward BD, Bezanson KD, Hillyer LM, Lee WH. The CD45RA+ (quiescent) cellular phenotype is overabundant relative to the CD45RA- phenotype within the involuted splenic $\mathrm{T}$ cell population of weanling mice subjected to wasting protein-energy malnutrition. J Nutr. 1995;125(10):2471-2482.

3. Fritsche KL, Anderson M, Feng C. Consumption of eicosapentaenoic acid and docosahexaenoic acid impair murine interleukin-12 and interferon-gamma production in vivo. J Infect Dis. 2000;182 Suppl 1:S54-61.

4. Azzara A, Carulli G, Sbrana S, Rizzuti-Gullaci A, Minnucci S, Natale M, Ambrogi F. Effects of lysine-arginine association on immune functions in patients with recurrent infections. Drugs Exp Clin Res. 1995;21(2):71-78.

5. Bansal V, Ochoa JB. Arginine availability, arginase, and the immune response. Curr Opin Clin Nutr Metab Care. 2003;6(2):223-228.

6. Barbul A. Arginine and immune function. Nutrition. 1990;6(1):53-58; discussion 59-62.

7. Bronte V, Zanovello P. Regulation of immune responses by L-arginine metabolism. Nat Rev Immunol. 2005;5(8):641-654.

8. Chuntrasakul C, Siltharm S, Sarasombath S, Sittapairochana C, Leowattana W, Chockvivatanavanit S, Bunnak A. Metabolic and immune effects of dietary arginine, glutamine and omega-3 fatty acids supplementation in immunocompromised patients. J Med Assoc Thai. 1998;81(5):334-343.

9. Cynober L. Immune-enhancing diets for stressed patients with a special emphasis on arginine content: analysis of the analysis. Curr Opin Clin Nutr Metab Care. 2003;6(2):189-193.

10. Droge W. Oxidative stress and ageing: is ageing a cysteine deficiency syndrome? Philos Trans R Soc Lond B Biol Sci. 2005;360(1464):2355-2372.

11. Grimble RF. Nutritional modulation of immune function. Proc Nutr Soc. 2001;60(3):389-397.

12. Hamani D, Charrueau C, Butel MJ, Besson V, Belabed L, Nicolis I, Le Plenier S, et al. Effect of an immune-enhancing diet on lymphocyte in head-injured rats: what is the role of arginine? Intensive Care Med. 2007;33(6):1076-1084.

13. Klausz G, Buzas E, Scharek P, Tiszlavicz L, Gyulai Z, Fulop AK, Falus A, et al. Effects of Helicobacter pylori infection on gastric inflammation and local cytokine production in histamine-deficient (histidine decarboxylase knock-out) mice. Immunol Lett. 2004;94(3):223228.

14. Suarez Butler MF, Langkamp-Henken B, HerrlingerGarcia KA, Klash AE, Szczepanik ME, Nieves C, Jr., Cottey RJ, et al. Arginine supplementation enhances mitogen-induced splenocyte proliferation but does not affect in vivo indicators of antigen-specific immunity in mice. J Nutr. 2005;135(5):1146-1150.

15. Spurlock ME. Regulation of metabolism and growth during immune challenge: an overview of cytokine 
function. J Anim Sci. 1997;75(7):1773-1783.

16. Borghetti AF, Kay JE, Wheeler KP. Enhanced transport of natural amino acids after activation of pig lymphocytes. Biochem J. 1979;182(1):27-32.

17. Grimm H, Kraus A. Immunonutrition--supplementary amino acids and fatty acids ameliorate immune deficiency in critically ill patients. Langenbecks Arch Surg. 2001;386(5):369-376.

18. Yoo SS, Field CJ, McBurney MI. Glutamine supplementation maintains intramuscular glutamine concentrations and normalizes lymphocyte function in infected early weaned pigs. J Nutr. 1997;127(11):2253-2259.

19. Lin MT, Kung SP, Yeh SL, Lin C, Lin TH, Chen KH, Liaw KY, et al. The effect of glutamine-supplemented total parenteral nutrition on nitrogen economy depends on severity of diseases in surgical patients. Clin Nutr. 2002;21(3):213-218.

20. Roebothan BV, Chandra RK. Relationship between nutritional status and immune function of elderly people. Age Ageing. 1994;23(1):49-53.

21. Reade MC, Clark MF, Young JD, Boyd CA. Increased cationic amino acid flux through a newly expressed transporter in cells overproducing nitric oxide from patients with septic shock. Clin Sci (Lond). 2002;102(6):645650 .

22. Wilmore DW. The effect of glutamine supplementation in patients following elective surgery and accidental injury. J Nutr. 2001;131(9 Suppl):2543S-2549S; discussion 2550S-2541S.

23. Calcagni E, Elenkov I. Stress system activity, innate and T helper cytokines, and susceptibility to immune-related diseases. Ann N Y Acad Sci. 2006;1069:62-76.

24. Elenkov IJ, Chrousos GP. Stress hormones, proinflammatory and antiinflammatory cytokines, and autoimmunity. Ann N Y Acad Sci. 2002;966:290-303.

25. Elenkov IJ, Chrousos GP. Stress hormones, Th1/ Th2 patterns, pro/anti-inflammatory cytokines and susceptibility to disease. Trends Endocrinol Metab. 1999;10(9):359-368.

26. Matalka KZ. Neuroendocrine and cytokines-induced responses to minutes, hours, and days of mental stress. Neuro Endocrinol Lett. 2003;24(5):283-292.

27. Bauer ME, Vedhara K, Perks P, Wilcock GK, Lightman SL, Shanks N. Chronic stress in caregivers of dementia patients is associated with reduced lymphocyte sensitivity to glucocorticoids. J Neuroimmunol. 2000;103(1):8492.

28. Maes M, Meltzer HY, Stevens W, Cosyns P, Blockx P. Multiple reciprocal relationships between in vivo cellular immunity and hypothalamic-pituitary-adrenal axis in depression. Psychol Med. 1994;24(1):167-177.

29. Molijn GJ, Spek JJ, van Uffelen JC, de Jong FH, Brinkmann AO, Bruining HA, Lamberts SW, et al. Differential adaptation of glucocorticoid sensitivity of peripheral blood mononuclear leukocytes in patients with sepsis or septic shock. J Clin Endocrinol Metab. 1995;80(6):17991803.

30. Glaser R, Kutz LA, MacCallum RC, Malarkey WB. Hormonal modulation of Epstein-Barr virus replication. Neuroendocrinology. 1995;62(4):356-361.

31. Uchakin PN, Gotovtseva EP, Stray-Gundersen J. Immune and endocrine alterations in marathon runners. J Appl Res. 2003;3(4):483-494.

32. Uchakin PN, Stowe RP, Paddon-Jones D, Tobin BW, Ferrando AA, Wolfe RR. Cytokine secretion and latent herpes virus reactivation with 28 days of horizontal hypokinesia. Aviat Space Environ Med. 2007;78(6):608612.

33. Feinstein MB, Schleimer RP. Regulation of the action of hydrocortisone in airway epithelial cells by 11 betahydroxysteroid dehydrogenase. Am J Respir Cell Mol Biol. 1999;21(3):403-408.

34. Whorwood CB, Sheppard MC, Stewart PM. Licorice inhibits 11 beta-hydroxysteroid dehydrogenase messenger ribonucleic acid levels and potentiates glucocorticoid hormone action. Endocrinology. 1993;132(6):22872292.

35. Tobin BW, Leeper-Woodford SK, Hashemi BB, Smith SM, Sams CF. Altered TNF-alpha, glucose, insulin, and amino acids in islets of Langerhans cultured in a microgravity model system. Am J Physiol Endocrinol Metab. 2001;280(1):E92-102.

36. Uchakin PN, Tobin BW, Morukov BV, Larina IV, Cubbage ML. Type 1 vs. type 2 cytokine secretion in vitro and its regulation by hydrocortisone in humans subjected to 120-day anti-orthostatic bed-rest regime. J Gravit Physiol. 2002;9(2):71-82.

37. Awad S, Yokozeki H, Miyazaki Y, Igawa K, Minatohara K, Satoh T, Nishioka K. Glucocorticoids induced the production and gene expression of IL-1alpha through AP-1 and partially NF-kappaB activation in murine epidermal cells. J Med Dent Sci. 2002;49(1):27-35.

38. Janele D, Lang T, Capellino S, Cutolo M, Da Silva JA, Straub RH. Effects of testosterone, 17beta-estradiol, and downstream estrogens on cytokine secretion from human leukocytes in the presence and absence of cortisol. Ann N Y Acad Sci. 2006;1069:168-182.

39. Jones HN, Ashworth CJ, Page KR, McArdle HJ. Cortisol stimulates system A amino acid transport and SNAT2 expression in a human placental cell line (BeWo). Am J Physiol Endocrinol Metab. 2006;291(3):E596-603.

40. Miyazaki Y, Yokozeki H, Awad S, Igawa K, Minatohara K, Satoh T, Katayama I, et al. Glucocorticoids augment the chemically induced production and gene expression of interleukin-1alpha through NF-kappaB and AP-1 activation in murine epidermal cells. J Invest Dermatol. 2000;115(4):746-752.

41. Harimaya A, Tarkkanen J, Mattila P, Fujii N, Ylikoski J, 
Himi T. Difference in cytokine production and cell activation between adenoidal lymphocytes and peripheral blood lymphocytes of children with otitis media. Clin Diagn Lab Immunol. 2005;12(9):1130-1134.

42. Reid IR, Katz JM, Ibbertson HK, Gray DH. The effects of hydrocortisone, parathyroid hormone and the bisphosphonate, APD, on bone resorption in neonatal mouse calvaria. Calcif Tissue Int. 1986;38(1):38-43.

43. Al-Tamemi S, Zuzan H, Norris A, Saenz A, Ponton A, Guay J, et al. Hydrocortisone upregulates Th2-type cytokine and chemokine receptor mRNA on human B-lymphocytes. J Allergy Clin Immunol. 2006;117(2 Suppl AAAAI 62ND):S245.

44. Flynn NE, Wu G. Enhanced metabolism of arginine and glutamine in enterocytes of cortisol-treated pigs. Am J Physiol. 1997;272(3 Pt 1):G474-480.

45. Jones HN, Jansson T, Powell TL. IL-6 stimulates system A amino acid transporter activity in trophoblast cells through STAT3 and increased expression of SNAT2. Am J Physiol Cell Physiol. 2009;297(5):C1228-1235. 